\title{
Determinantes da Propensão para Inovar e da Intensidade Inovativa em Empresas da Indústria de Alimentos do Brasil
}

\author{
José Ednilson de Oliveira Cabral
}

\section{RESUMO}

Este artigo tem por objetivo identificar as variáveis organizacionais que determinam a probabilidade e intensidade de inovações em empresas da indústria de alimentos do Brasil. A partir das propostas de analisar ativos (recursos e competências) e integração destes como determinantes da geração e adoção de inovações tecnológicas, uma estrutura conceitual foi definida para identificar as variáveis ligadas à probabilidade de uma empresa inovar e daquelas que afetam a maior intensidade inovativa, uma vez que a empresa tenha inovado. Uma abordagem double-hurdle envolvendo modelos censurado e truncado foi utilizada. Nos testes dos modelos foram utilizados os dados de um levantamento sobre o processo de inovação tecnológica em uma amostra de 248 empresas. Os resultados confirmaram que os ativos para inovação 'investimento em tecnologia externamente desenvolvida, investimento em $P \& D$ executado internamente e alianças com outras empresas, institutos de pesquisa e universidades para execução externa de $\mathrm{P} \& \mathrm{D}$, juntamente com tamanho de empresa' determinam significativamente a probabilidade de uma empresa inovar. Por sua vez, as variáveis 'alianças com outras empresas, institutos de pesquisa e universidades para execução externa de $\mathrm{P} \& \mathrm{D}$, tamanho de empresa e orientação para o mercado' foram associadas com a intensidade inovativa na indústria.

Palavras-chave: inovações; ativos para inovar; ativos complementares; indústria brasileira de alimentos; economia da inovação.

\begin{abstract}
This paper defines the relative role of firms' characteristics on both their likelihood to innovate and the intensity of innovations. Specifically, on the basis of a survey of food firms, it identifies which innovative and complementary assets, when combined in a multivariate framework, are firstly statistically significant in distinguishing innovating from non-innovating firms in the Brazilian Food Industry (BFI), and secondly in distinguishing more from less innovating firms. The objective requires that the analytical model should be adequate to explain firstly the differences observed between an individual firm's likelihood to innovate, and secondly the differences in innovation intensity between innovating firms. Thus, a double-hurdle approach involving censored and truncated models was applied. The findings confirm that firms' innovative assets investment in external technology, investment in $\mathrm{R} \& \mathrm{D}$, and external alliances, alongside firm size are the significant variables in determining firms' likelihood to innovate, whereas external alliances, firm size and market orientation are related to intensity to innovate in the BFI.
\end{abstract}

Key words: innovation; innovative assets; complementary assets; Brazilian food industry; economics of innovation. 


\section{INTRODUÇÃO}

Desde tempos ancestrais, os seres humanos têm baseado suas atividades em conhecimento técnico. Nesse sentido, mudanças na tecnologia tem desempenhado papel fundamental na elevação do padrão de vida da humanidade. Um bom exemplo pode ser tirado das mudanças na preparação de alimentos após a descoberta do fogo. No entanto, é geralmente reconhecido que a lentidão na transformação tecnológica no passado foi substituída por uma acelerada corrente de mudanças após a revolução industrial inglesa no século XVIII, a ponto de esse período ter sido chamado de 'a era da tecnologia'.

Toffler (1970) afirmou que a tecnologia é não apenas a fonte de mudança, mas também o motor dela. Para ilustrar isto, um exemplo clássico é dado pela produção de agulhas, cuja fábrica foi exemplo de eficiência estática para Adam Smith (1776). A produtividade média de 4.800 agulhas por trabalhador/dia alcançada nos anos 1770 foi elevada, dois séculos depois, para mais de 800.000 , graças a inúmeras mudanças tecnológicas ${ }^{(1)}$.

Portanto, ainda que considerando as externalidades negativas de tecnologias, ninguém deve, como sugerido por Freeman e Soete (1997), ignorar o impacto dos benefícios das mudanças tecnológicas sobre nossas vidas diárias. Para estes autores, "no mundo da microeletrônica e engenharia genética, é desnecessário enfatizar a importância da ciência e tecnologia para a economia" (p.1).

Mudança tecnológica, em sentido estrito, refere-se a processos de produção novos ou aperfeiçoados e à criação ou melhoria de produtos. Schumpeter (1939) propôs uma útil tipologia analítica, classificando esta mudança em três estágios: invenção, inovação e imitação (difusão). Invenção é o estágio que corresponde a geração de novas idéias, desenho, modelo ou protótipos. Difusão é o estágio pelo qual processos e produtos se disseminam pela economia. $\mathrm{O}$ objeto deste trabalho é o segundo estágio, inovação, que se refere ao processo que traduz uma nova idéia em produtos ou processos comercializáveis pela primeira $\mathrm{vez}^{(2)}$.

Em nível de empresa, "inovação pode ser reconhecida como a principal fonte de vantagem competitiva" (Grunert et al., 1995, p. 4). Schumpeter (1939) sugere que inovação é o principal motor do desenvolvimento capitalista e fonte de lucro empresarial. Em breve, inovação pode ser considerada como uma das principais forças direcionadoras do desenvolvimento econômico e também da competitividade empresarial ${ }^{(3)}$. 
Devido aos seus impactos e efeitos, pesquisadores de economia e administração têm, a partir dos anos 1950, crescentemente elevado seus interesses pelas causas ou determinantes da inovação tecnológica. O objetivo fundamental tem sido ampliar as bases fatuais para teorias e políticas. Consequientemente, um crescente número de estudos tem sido realizados com o intuito de identificar quais os fatores que influenciam ou determinam a atividade inovativa em níveis de indústria e/ou empresa. Apesar disto, os resultados dessas pesquisas têm sido inconsistentes ou contraditórios. Além do mais, não existem estudos específicos sobre a Indústria Brasileira de Alimentos (IBA na sequência). Portanto, a questão dos determinantes da inovação nesta indústria, constituem o problema deste estudo.

Adianta-se que o trabalho se concentra nos determinantes microeconômicos da inovação, utilizando contribuições conceituais da economia, tecnologia e organização. Esta concentração significa que importantes fatores macroeconômicos ou do contexto da empresa, tais como políticas fiscais ou incentivos governamentais, não são avaliados. Pela mesma razão, não são avaliadas as conseqüências das inovações sobre o sistema econômico.

Os resultados dos trabalhos de pesquisa sobre o assunto indicam que a atividade inovativa é um processo complexo e diversificado. No entanto, eles também indicam que o processo não é aleatório ou imprevisível e que ele é específico da firma ou da indústria. Portanto, considerando a importância da inovação para o crescimento econômico e competitividade empresarial, é de fundamental importância conhecer os fatores que influenciam ou determinam a atividade inovativa dentro das empresas e indústrias e entre elas. A ausência ou limitação de tal entendimento torna menos eficiente aspectos como a escolha tecnológica em empresas, a definição de políticas tecnológicas por governos e o desenvolvimento de estruturas preditivas por analistas.

Assim, o objetivo geral deste artigo é determinar o papel relativo de características de empresas e da indústria sobre a probabilidade de uma empresa inovar na IBA. Especificamente, este objetivo refere-se à identificação, através de análise multivariada, dos ativos para inovação e dos ativos complementares que são estatisticamente significativos em distinguir empresas inovativas das não-inovativas e em distinguir as empresas mais das menos inovativas na IBA.

Além desta introdução, este artigo apresenta quatro seções. A primeira, revisa em profundidade a literatura sobre ativos (recursos e capacidades) e ligações entre ativos utilizados na produção e exploração comercial de inovações tecnológicas com a finalidade de estabelecer o modelo conceitual para realização do objetivo. Nesta seção são formuladas as hipóteses do estudo. Na seção 2 é apresentada a metodologia da pesquisa, compreendendo a base de dados, o método 
e o modelo analítico. Na terceira seção os resultados da pesquisa são apresentados e discutidos; quarta seção encerra o estudo com as considerações finais.

\section{Ativos Inovativos e Complementares e Inovação Tecnológica Na Indústria Brasileira de Alimentos (IBA)}

As propostas de Christensen $(1995,1996)$ e Teece (1996) de analisar ativos (recursos e capacidades) e ligações entre esse ativos com a finalidade de produzir e explorar comercialmente inovações tecnológicas, resultam na estrutura conceitual apresentada na Figura 1.

Figura 1: Determinantes da Inovação Tecnológica

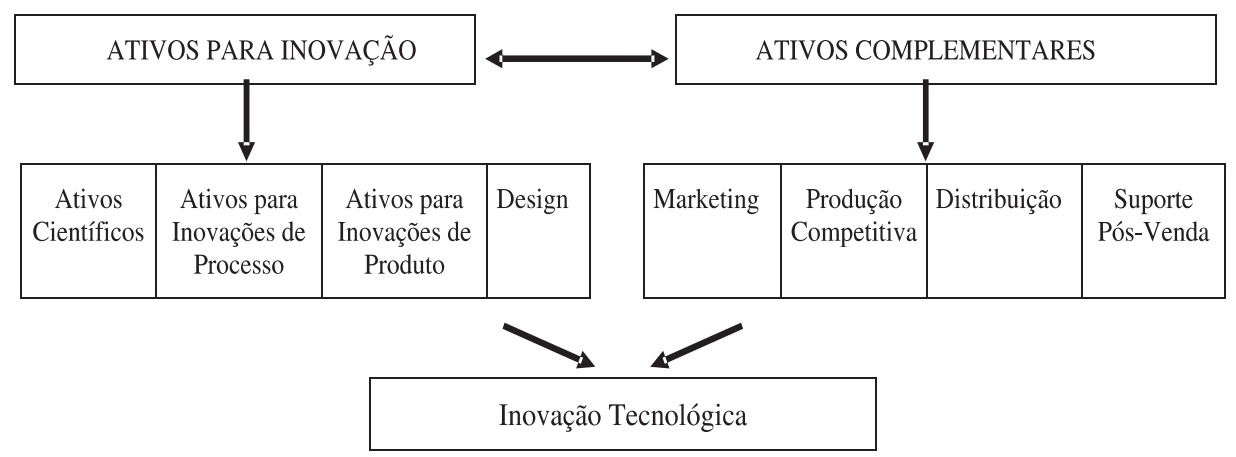

Dessa estrutura, Christensen (1995, p. 730) destaca a importância da necessidade de se dispor de relevantes ativos para inovação, com a finalidade de se assegurar a produção de inovações tecnológicas. O ponto básico da abordagem é que as inovações tecnológicas requerem não apenas a aplicação do esforço em pesquisa e desenvolvimento (P\&D), mas também (algumas vezes até com a exclusão de P\&D) a utilização de diferentes tipos de ativos inovativos para desenvolvimento de inovações. Christensen (1996, p. 197) propõe que P\&D é um conceito limitado para indicar plenamente a amplitude de ativos inovativos necessários à inovação tecnológica. Em geral, o conceito de P\&D dentro de empresas está restrito ao que "acontece no departamento de P\&D ou de engenharia da empresa" e "no seu estreito significado estatístico, grosseiramente subestima ou até negligencia partes importantes do esforço de desenvolvimento tecnológico" (Christensen, 1996, p. 196).

Portanto, os ativos para inovação, contemplados nessa abordagem, compreendem os recursos e capacidades "necessários à produção de novas ou 
aperfeiçoadas tecnologias, isto é, ao desenvolvimento de novos produtos ou processos" (Christensen, 1996, p. 197). Estes ativos estão divididos em quatro grupos genéricos: ativos para a pesquisa científica; ativos para inovação de processo; ativos para inovação de produto; e ativos relativos ao design estético. Eles foram definidos por Christensen (1996, p. 198) conforme abaixo:

a. Ativos para a pesquisa científica (correspondendo ao P em P\&D) envolvem tanto a pesquisa básica de natureza pré-competitiva e aplicada, quanto a pesquisa industrial que fornece os insumos diretos para utilização no desenvolvimento de processos e novos produtos.

b. Ativos para inovação de processo compreendem tanto os recursos e capacidades tangíveis (hardware) utilizados para as inovações de processo, quanto as capacidades sistêmicas (tácitas) envolvidas no desenvolvimento de sistemas de produção, logísticas interior e exterior, controle de qualidade e layout.

c. Ativos para inovação de produto são os recursos e capacidades (excluídos os tipos descritos em ' $a$ ' e 'd') necessários ao desenvolvimento de produtos, tais como: engenharia de produto, instrumentação e desenvolvimento de software.

d. Ativos relativos ao design estético são comumente considerados como parte da função de marketing de desenvolvimento de produtos; mas eles também podem ser considerados como uma parte distinta, cuja principal função é fazer a ponte entre as características técnicas e funcionais do produto com os atributos de marketing.

Essas categorias de ativos para a inovação representam um modelo compreensivo para entendimento da base multidimensional do processo de inovação dentro das empresas. Enquanto em algumas empresas o processo é mais concentrado na pesquisa formal, em outras predominam formas diversas de aprendizagem interna ou design estético. Porém a maioria das empresas utiliza mais de uma categoria de ativo. Christensen $(1995,1996)$ inspirou-se no framework desenvolvido pela economia dos custos de transação, mais particularmente na análise da especificidade de ativos, para avaliar e caracterizar o grau pelo qual certas atividades inovativas baseadas em um tipo de ativo dependem de outro ou de outros ativos. Assim, o grau de especificidade entre ativos é baixo, se a atividade inovativa se relaciona a apenas um tipo de ativo. $\mathrm{O}$ grau pode ser considerado alto, se existir a conexão de dois ou mais tipos de ativos, como pode ser exemplificado por uma situação em que uma inovação de produto requer alterações radicais no processo de produção e/ou foi baseada em atividades prévias de pesquisa científica. Neste artigo, três categorias de indicadores de ativos para inovação serão avaliadas: 
1) investimento em $P \& D$; 2) contratos externos, objetivando o desenvolvimento de inovações; e 3) investimentos em tecnologias desenvolvidas externamente à empresa.

Considerando que a proporção do investimento em $\mathrm{P} \& \mathrm{D}$ sobre vendas na indústria de alimentos, comparada a outras indústrias, é relativamente baixa, esta atividade de pesquisa geralmente não é considerada como importante ativo para inovação na referida indústria. No entanto, a emergência de novos 'paradigmas tecnológicos' para a indústria de processamento de alimentos tem motivado empresas do setor a desenvolverem pesquisas internamente ou contratarem o desenvolvimento através de empresas ou instituições independentes, elevando de um lado seus gastos na atividade de $\mathrm{P} \& \mathrm{D}$ e de outro sua capacidade tecnológica. Além do mais, em termos gerais, Cohen e Levinthal (1989) atribuíram um duplo papel para $\mathrm{P} \& \mathrm{D}$, qual seja, o de que esta atividade é importante não apenas para a inovação, mas também para elevar a capacidade da empresa em assimilar e explorar novos conhecimentos. Portanto, desde que a atividade de inovação, em qualquer forma, demanda competência por parte da empresa, a atividade de $\mathrm{P} \& \mathrm{D}$ desempenha importante papel na incorporação da competência necessária para desenvolver, assimilar e adaptar tecnologias.

Conseqüentemente, as proposições teóricas sobre o papel da P\&D na inovação das empresas alimentícias são ambíguas. No entanto, trabalhos empíricos já realizados têm encontrado forte relacionamento entre inovações e P\&D nas indústrias em geral, mas o resultado não se repete para a indústria de alimentos em particular (exemplo Connor, Rogers, Marion, \& Muelle, 1985). Por sua vez, dadas as proposições de que o esforço em P\&D eleva a competência inovativa de uma empresa, pode-se hipotetizar que existe relacionamento significativo entre investimentos em $P \& D$ e intensidade inovativa nas empresas. Adicionalmente, desde que uma enorme parcela dos gastos com P\&D, por parte das empresas alimentícias, é alocada para contratar externamente a atividade de pesquisa, podese hipotetizar também que é significativo o relacionamento entre alianças (contratos) com outras empresas, institutos de pesquisa e universidades para execução externa de $\mathrm{P} \& \mathrm{D}$ e inovação nas empresas.

Com relação ao nível de investimento em tecnologias desenvolvidas externamente à empresa, existem proposições (exemplos Connor et al., 1985; Galizzi \& Venturini, 1996) de que a elevação da eficiência técnica e o alto nível de crescimento de produtividade observados na indústria de alimentos são explicados pelas inovações de processo desenvolvidas em outras indústrias.

Do exposto são formuladas as seguintes hipóteses sobre o relacionamento entre ativos para a inovação e produção de inovações na IBA: 
H1. Considerando a predominância de inovações incrementais e/ou relacionadas a investimentos de capital, os investimentos com $P \& D$ não apresentam forte relacionamento com a propensão de uma empresa alimentícia a inovar.

H2. Considerando que a atividade de P\&D eleva a capacidade de a empresa vir a inovar, o investimento nela é fortemente relacionado com a intensidade inovativa de empresas na IBA.

H3. Considerando a importância de P\&D na elevação do grau de inovação e o nível de terceirização dessa atividade na indústria de alimentos, alianças com outras empresas, institutos de pesquisa e universidades para a execução externa de P\&D estão relacionadas com a propensão para inovar na IBA.

H4. Dado o alto nível de investimentos em tecnologias desenvolvidas externamente à IBA, são significativos os relacionamentos deste investimento tanto com a propensão para inovar, quanto com a intensidade de inovações nas empresas alimentícias.

A estrutura conceitual proposta por Christensen, acima descrita, está concentrada na análise dos ativos inovativos requeridos para a produção de inovações tecnológicas. Porém a atividade inovativa apenas se completa, quando a inovação é comercializada com sucesso. Na realização desta etapa, empresas inovativas também precisam dos ativos complementares exigidos para apoiar a atividade inovativa e garantir os benefícios da inovação (Teece, 1986). Estes ativos complementares relacionam-se com as atividades típicas de áreas funcionais das empresas, tais como marketing, produção competitiva, distribuição e suporte pósvenda. Estes ativos tanto podem ser internalizados, quanto contratados a jusante da cadeia produtiva.

Desta forma, vários estudos têm objetivado definir o papel relativo de ativos complementares selecionados sobre a inovatividade de empresas. As variáveis utilizadas para mensurar este papel incluem o grau de orientação para o mercado, o nível de integração vertical e o nível de diversificação horizontal das empresas. Em relação à orientação para o mercado, foram selecionados como indicadores a influência dos gastos com propaganda e intensidade de exportações sobre inovações ${ }^{(4)}$. Propaganda é considerada atividade de marketing muito importante no suporte das inovações na indústria de alimentos, particularmente no caso de inovações de produto. Adicionalmente, Galizzi e Venturini (1996, p. 142) verificaram que esta importância também depende do cliente-alvo. Por sua vez, a importância das exportações para inovação também é relativa, dependendo, entre outros aspectos, do tamanho e do potencial do mercado doméstico das empresas. No caso da indústria brasileira em geral, Braga e Wilmore (1991, p. 429) verificaram que as exportações influenciam positivamente os esforços 
tecnológicos. Porém os autores anteciparam que este resultado varia de uma indústria para outra.

Com relação à integração vertical, propõe-se em geral que o nível mais elevado de integração favorece uma maior intensidade inovativa. Porém este relacionamento é mediado por uma série de outras variáveis, tais como especificidade dos ativos, tipo de inovação (autônoma ou sistêmica ${ }^{(5)}$ ) e nível de apropriabilidade tecnológica. As proposições predominantes são de que a integração vertical favorece a inovação, quando existe alto nível de especificidade de ativos, predominância de inovações sistêmicas e baixo nível de apropriabilidade. Portanto, considerando os aspectos prevalecentes das inovações na indústria de alimentos, tais como incrementais, de processo e novas apenas para a empresa inovativa (imitação), pode-se considerá-las como autônomas e apresentando pouca apropriabilidade. Por outro lado, estima-se que enquanto a inovação autônoma favorece a descentralização, a baixa apropriabilidade favorece a integração. Desta forma, não se pode apresentar afirmações prévias sobre o relacionamento entre integração vertical e inovatividade. No entanto, como o nível geral de especificidade dos ativos é baixo na indústria de alimentos, este relacionamento não deve ser significativo.

Por sua vez, razões são apresentadas (a exemplo de Teece, 1996, p. 203) para sugerir que o desempenho inovativo é impactado pelo escopo das empresas. Sugere-se também (a exemplo de Nelson, 1959) que o efeito da diversificação horizontal sobre intensidade inovativa deve ser mais significativo, quando associado à pesquisa básica. Este não é, definitivamente, o caso da indústria de alimentos.

Das proposições acima sobre o relacionamento entre ativos complementares e inovatividade, as seguintes hipóteses são formuladas:

H5. Dada a predominância de inovações de processo na IBA, o nível de gastos com propaganda não está significativamente relacionado à propensão para inovar na indústria.

H6. Considerando que a intensidade de inovações na indústria está ligada com uma maior proporção de inovações de produto, o nível de gastos com propaganda está significativamente associado com a intensidade inovativa das empresas.

H7. Dados o tamanho e o potencial de crescimento do mercado brasileiro de alimentos, não existe associação significativa entre nível de exportações e inovatividade na IBA (propensidade e intensidade).

H8. Níveis mais elevados de integração vertical e diversificação horizontal não determinam maiores propensidade e intensidade de inovação na IBA. 
A seguir, será analisado o efeito de variáveis complementares sobre o processo inovativo na indústria de alimentos. O objetivo é avaliar o efeito dos ativos complementares sobre inovações, mediado pela influência de variáveis também consideradas como determinantes da atividade inovativa. São elas: tamanho de empresa e idade.

Tamanho tem sido uma das variáveis explanatórias mais testadas nos estudos sobre determinantes de atividade inovativa. Tanto as proposições teóricas quanto os resultados empíricos sobre este relacionamento não se têm revelado conclusivos. No último caso, é geralmente aceito que os resultados indefinidos estão ligados ao fato de que a relação depende do contexto, da indústria ou do setor econômico. Existem condições e indústrias cuja eficiência inovativa é prerrogativa de empresas menores e existem também outras condições e indústrias em que prevalece a inovação nas grandes empresas. No caso da indústria de alimentos, a maioria dos estudos empíricos tem confirmado a hipótese de que as maiores empresas são mais inovativas (a exemplos de Galizzi \& Venturini, 1996, p. 144; Huiban \& Boushina, 1998). Estes últimos autores também verificaram que o tamanho é o mais forte determinante da propensão para inovar em empresas da indústria de alimentos da França. Verificaram, ainda, que nesta indústria "a melhor situação para a inovação parece ser a de uma grande empresa atuando em um mercado não muito concentrado" (p. 396). No caso da indústria brasileira em geral, Braga e Wilmore (1991, p. 429) também verificaram que tamanho está significativamente relacionado a esforços tecnológicos, embora apresentando baixos coeficientes de estimação.

Com relação à idade de empresas, tem-se sugerido que a aprendizagem cumulativa favorece o desempenho inovativo. As hipóteses relacionadas às variáveis complementares são formuladas abaixo:

H9. As grandes empresas na IBA apresentam-se mais inovativas, tanto em propensão quanto em intensidade.

H10. As empresas mais antigas da IBA apresentam maior propensão e intensidade de inovações.

\section{Metodologia da Pesquisa}

As bases empíricas para a realização do objetivo deste artigo originam-se de uma base de dados criada a partir de uma pesquisa de campo, focalizando a atividade inovativa na IBA. Esta pesquisa foi definida visando a uma ampla 
descrição do processo inovativo e das características organizacionais das empresas na IBA.

A pesquisa mensurou inovação através de um indicador (proxy) direto, que tem sido aceito como indicação mais confiável, comparado ao uso de estatísticas sobre P\&D e patentes, da atividade inovativa dentro das empresas e entre indústrias ${ }^{(6)}$. Neste sentido, inovação foi definida como a primeira transação comercial de uma idéia que envolve um produto ou processo de produção, novo ou melhorado (Galizzi \& Venturini, 1996, p. 7). Portanto, inovação tecnológica envolve mudanças em processo de produção (inovação de processo) e a criação ou melhoria de um novo bem de consumo ou de capital (inovação de produto). Em alguns estudos, 'primeiro' tem sido qualificado como novo para o mundo ou país estudado (exemplo Pavitt, 1984, p. 344); em outros tem sido definido como novo para o mundo, país ou empresa (exemplo Cesaratto \& Mangano, 1993, p. 115; Christensen, Rama, \& Tunzelmann, 1996, p. 8; Myers \& Marquis, 1969, p. 3). Esta pesquisa seguiu essa última tendência, mensurando inovação em sentido amplo de novo para o mundo, país ou empresa ${ }^{(7)}$. Conseqüentemente, o indicador direto escolhido foi 'número de inovações', definido pela quantidade de inovações tecnológicas geradas e/ou adquiridas pelas empresas pesquisadas em um período de tempo previamente definido. Nas pesquisas até então realizadas, tem-se identificado estas inovações pedindo-se aos executivos para listar as inovações implementadas em suas empresas ou colocando o mesmo pedido para especialistas técnicos e observadores empresariais para indicarem as inovações implementadas em determinada linha de negócio ou setor econômico. Esta pesquisa mesclou as duas abordagens: primeiro, as empresas foram solicitadas a listar as inovações relativas aos três anos anteriores à pesquisa; segundo, a lista de inovações foi submetida a uma comissão de especialistas da Embrapa Empresa Brasileira de Pesquisa Agropecuária, para avaliar não apenas se a inovação informada deveria ser considerada como tal, mas também avaliar as características qualitativas informadas, em termos de complexidade, tipo e novidade da inovação (exemplos: radical versus incremental; produto ou processo). O principal resultado alcançado foi a padronização das respostas qualitativas.

Para a pesquisa, a indústria de processamento de alimentos foi definida tendo por base a classificação oficial do Governo brasileiro ${ }^{(8)}$. Por esta classificação, a indústria de alimentos é uma das 21 indústrias manufatureiras no grupo de dois dígitos e se divide em nove setores (nível de três dígitos). A partir desta definição, a relação de empresas na referida indústria foi adotada como base para seleção da amostra. Portanto, 38.916 empresas $^{(9)}$ constituíram a população $^{-}$ inicial. Como algumas questões foram consideradas grandes novidades para as empresas e no conjunto requeriam um arquivo interno muito organizado, as 
menores empresas (menos que cinco empregados) foram excluídas da população base. Portanto, a população definitiva foi composta por 19.045 empresas que representam $48,9 \%$ do total, porém respondem por $92,4 \%$ do emprego, $96,2 \%$ da folha de pagamento, $97,9 \%$ da produção e $97,6 \%$ do valor adicionado pela indústria.

A partir da população definitiva, uma amostra aleatória relativamente grande e estratificada de 1.000 empresas foi selecionada. A estratificação foi realizada com base na atividade econômica (setores de três dígitos), tamanho de empresa (número de empregados) e região geográfica do Brasil. Estas empresas foram solicitadas a preencher um questionário com informações sobre suas características organizacionais e atividades administrativas, estratégicas e inovativas, durante um período de três anos anteriores à pesquisa. $\mathrm{O}$ período de três anos foi definido para minimizar os efeitos de flutuações nas atividades inovativas anuais em empresas e na indústria e tendo em conta o fato de que inovação tecnológica não é um evento comum (bastante repetido) em nível de empresa. De fato, quanto mais longo o período, melhor a informação, especialmente para estudos longitudinais. No entanto, definiu-se o período de três anos para relacionar o início da pesquisa (1994) com o início de profunda reestruturação e início de estabilidade na economia brasileira.

Da amostra, 248 empresas $(24,8 \%)$ retornaram o questionário respondido. Destas, $167(67,4 \%)$ informaram que não inovaram no período da pesquisa, 77 $(31,0 \%)$ informaram que implementaram 233 inovações, quatro $(1,6 \%)$ não responderam à questão. Seis empresas foram excluídas da pesquisa; três por não corresponderem à definição de empresa processadora de alimentos (duas exclusivamente no setor de restaurante e uma exclusivamente na distribuição) e três porque não atenderam à solicitação por carta de esclarecer ou complementar determinadas informações. Das 233 inovações, a comissão de especialistas acima referida excluiu 95 por não se enquadrarem na definição oferecida de inovação tecnológica. Elas referiam-se a inovações organizacionais ou mudanças adaptativas na manufatura (simples alteração no processo de produção ou substituição de equipamento imprestável) ou simples variação de produto (mudanças na forma comercial, rótulo ou desenho da embalagem). Assim, 242 empresas, 66 empresas inovativas e 138 inovações forneceram a base para a análise realizada neste estudo. A distribuição destes números por setor da indústria (três dígitos) é apresentada na Tabela 1. 


\section{Tabela 1: Setores da Indústria de Alimentos do Brasil e Inovatividade}

\begin{tabular}{|c|c|c|c|c|c|c|c|c|}
\hline \multirow{2}{*}{$\begin{array}{l}\text { SETOR } \\
\text { Processamento de Carnes }\end{array}$} & \multicolumn{2}{|c|}{$\begin{array}{l}\text { EMPRESAS PESQ } \\
(\mathrm{N}) \quad(\% \mathrm{PEP})\end{array}$} & \multicolumn{3}{|c|}{$\begin{array}{l}\text { EMPRESAS INOVATIVAS } \\
\text { (N) } \% \text { PEI) (INDEX) }\end{array}$} & \multicolumn{3}{|c|}{$\begin{array}{l}\text { INOVAÇõES } \\
\text { (N) (\%PIT) (INDEX) }\end{array}$} \\
\hline & & & & & & & & \\
\hline Peixes & 32 & 13.2 & 8 & 12.1 & .92 & 18 & 13.1 & .99 \\
\hline Frutas e Vegetais & 19 & 7.9 & 7 & 10.6 & 1.34 & 22 & 15.9 & 2.01 \\
\hline Gorduras e Óleos & 10 & 4.1 & 2 & 3.0 & .73 & 2 & 1.5 & .36 \\
\hline Laticínios & 27 & 11.2 & 6 & 9.1 & .81 & 10 & 7.2 & .64 \\
\hline Grãos e Ração Animal & 53 & 21.9 & 9 & 13.7 & .63 & 16 & 11.6 & .53 \\
\hline Açúcar & 13 & 5.4 & 5 & 7.6 & 1.41 & 18 & 13.1 & 2.43 \\
\hline Café & 9 & 3.7 & 2 & 3.0 & .81 & 5 & 3.6 & .97 \\
\hline \multicolumn{9}{|l|}{ Chocolates, Panificação, } \\
\hline Massas & 57 & 23.5 & 20 & 30.3 & 1.29 & 34 & 24.6 & 1.05 \\
\hline Outros & 22 & 9.1 & 7 & 10.6 & 1.16 & 13 & 9.4 & 1.03 \\
\hline TOTAL & 242 & 100 & 66 & 100 & - & 138 & 100 & - \\
\hline
\end{tabular}

$\mathrm{PEP}=$ Proporção das empresas pesquisadas; $\mathrm{PEI}=$ Proporção das empresas inovativas; $\mathrm{PIT}=$ Proporção do total de inovações; Index=PEI/PEP ou PIT/PEP. Índex maior que 1 significa que o setor apresenta um desempenho, em termos de empresas inovativas ou inovações, acima da média da indústria.

O modelo analítico a ser utilizado deverá ser adequado à identificação das variáveis que afetam a probabilidade ou propensão de uma empresa vir a inovar e também daquelas que influenciam um maior número de inovações (intensidade inovativa), uma vez a empresa tenha inovado. Um modelo bastante utilizado na literatura para atender a este duplo objetivo tem sido o modelo Tobit de regressão. Mas, como não existe razão para que as mesmas variáveis afetem as duas decisões acima (como se pode depreender das proposições teóricas acima revisadas), o uso de dois modelos analíticos ou a aplicação de uma abordagem double-hurdle tem sido geralmente sugeridos (exemplos Burton, Tomlinson, \& Young, 1994; Goetz, 1995; Wakelin, 1998). O primeiro deve ser um modelo censurado em que a variável dependente, propensão para inovar, é definida pela probabilidade de a empresa decidir inovar (codificado como $\mathrm{LI}_{\mathrm{i}}=1$ se a empresa alimentícia $\mathrm{I}^{\text {th }}$ inovar; ou $\mathrm{LI}_{i}=0$ se a empresa $\mathrm{I}^{\text {th }}$ não inovar). $\mathrm{O}$ segundo deve ser um modelo truncado, em que a variável dependente, intensidade inovativa ou produção de inovações é considerada apenas, se apresentar valor superior a zero (0). Esta variável é codificada como $\mathrm{PI}_{\mathrm{i}}>0$, com PI representando o número de inovações da empresa no período da pesquisa.

Com referência aos ativos inovativos, os indicadores incluem: A variável gastos em P\&D (redmo) foi mensurada pela proporção do faturamento das empresas investida na atividade, a qual constitui uma medida usual de intensidade de P\&D, a partir de sete classes colocadas no questionário. A variável alianças para 
execução externa de P\&D (altot) foi mensurada pelo número de contratos que as empresas na amostra assinaram, no período da pesquisa, com empresas privadas ou instituições públicas ou universidades para desenvolvimento de inovações tecnológicas. A variável atividades de inovação tecnológica (tecmo), excetuando gastos em P\&D, foi mensurada pela proporção do faturamento destinada à aquisição de tecnologia desenvolvida externamente, também a partir das sete classes colocadas no questionário.

Os indicadores dos ativos complementares incluem: propaganda (advmo) mensurada pela proporção de gastos com propaganda sobre faturamento (sete classes). Exportações (expmo) calculada pelo percentual do faturamento originado de exportações (sete classes). Integração vertical (proto) referiu-se ao número de estágios de produção e distribuição em que a empresa atuava na cadeia produtiva. Finalmente, diversificação horizontal (atota) foi mensurada pelo número de segmentos (subsetores no nível de quatro dígitos) que cada empresa produzia e/ou comercializava.

Como definido anteriormente, as variáveis complementares incluem tamanho e idade de empresa. Em termos de tamanho, a maioria dos estudos tem utilizado o total de empregados equivalentes em tempo integral na mensuração da variável. Porém alguns estudos (exemplo Scherer, 1965) têm preferido utilizar vendas anuais, uma vez que as proposições teóricas de influência do tamanho sobre inovatividade são geralmente baseadas na disponibilidade de recursos financeiros ou fluxo de caixa. No entanto, estas variáveis embora não perfeitamente correlacionadas, apresentam elevada correlação positiva em vários estudos. Assim, serão utilizados os dois indicadores: tamanho será mensurado em uma primeira equação por número de empregados (empme); alternativamente uma segunda equação será calculada com a variável representada por faturamento (turmo). Adicionalmente, muitos estudos têm demonstrado que a atividade inovativa se eleva com o tamanho da empresa, no entanto até determinado limite. Então, eles concluem que não existe linearidade na relação. Desta forma, uma especificação quadrática para as variáveis tamanho (empme e turmo $^{2}$ ) será incluída no modelo para teste de possível não linearidade. Finalmente, idade (fiold) será mensurada pelo número de anos desde a criação da empresa até o ano da pesquisa.

A forma funcional econométrica do primeiro modelo é:

$\mathrm{LI}_{\mathrm{i}}=\mathrm{f}$ (redmo, altot, tecmo, advmo, expmo, proto, atota, empme, fiold)

Como anteriormente definido, a variável dependente é do tipo binário, recebendo o valor de zero ou unidade, com predominância do primeiro. A especificação deve receber a forma de um dos modelos censurados, dos quais o modelo de regressão Logit é um dos mais utilizados. 
A forma funcional econométrica do segundo modelo é:

$\mathrm{PI}_{\mathrm{i}}=\mathrm{f}$ (redmo, altot, tecmo, advmo, expmo, proto, atota, empme, fiold)

Também, como definido antes, a variável dependente apresenta valor superior a zero.

\section{Propensão e Intensidade de Inovações na IBA}

Os resultados das estimativas dos parâmetros para propensão das empresas alimentícias inovarem e para a intensidade das inovações nelas, a partir dos dois modelos (Logit e Truncado), são apresentados na Tabela 2. Como antecipado, dado o alto coeficiente de correlação apresentado pelos indicadores (proxys) de tamanho, empme e turmo, ambos apresentaram significância e direção de relacionamentos com as variáveis dependentes (probabilidade de inovar e inensidade inovativa) bastante semelhantes, com significância preditiva levemente superior para os modelos, utilizando o indicador empme. Desta forma, apenas os resultados com este indicador serão apresentados e discutidos a seguir.

\section{Tabela 2: Parâmetros Estimados para Probabilidade e Intensidade Inovativa}

\begin{tabular}{|c|c|c|c|c|}
\hline \multirow{2}{*}{$\begin{array}{l}\text { VARIÁVEIS } \\
\text { EXPLANATÓRIAS }\end{array}$} & \multicolumn{2}{|c|}{ MODELO LOGIT } & \multicolumn{2}{|c|}{ MODELO TRUNCADO } \\
\hline & Equação 1 & Equação 2 & Equação 3 & Equação 4 \\
\hline Redmo & $0.47(0.24)^{* *}$ & $0.47(0.24)^{* *}$ & $-0.14(0.26)$ & $-0.20(0.20)$ \\
\hline Altot & $0.38(0.17)^{* *}$ & $0.39(0.18)^{* *}$ & $0.32(0.17)^{*}$ & $0.36(0.13)^{* * *}$ \\
\hline Tecmo & $1.10(0.26)^{* * *}$ & $1.10(0.26)^{* * *}$ & $-0.23(0.27)$ & $-0.84(0.20)$ \\
\hline Advmo & $0.2710(0.18)$ & $0.27(0.18)$ & $0.44(0.18)^{* *}$ & $0.32(0.13)^{* *}$ \\
\hline Expmo & $-0.20(0.22)$ & $-0.21(0.22)$ & $0.10(0.23)$ & $-0.16(0.18)$ \\
\hline Proto & $0.25(0.25)$ & $0.25(0.25)$ & $-0.29(0.32)$ & $-0.27(0.24)$ \\
\hline Atota & $-0.26(0.18)$ & $-0.26(0.18)$ & $-0.22(0.28)$ & $-0.28(0.21)$ \\
\hline Empme & 0.0019 & 0.002 & 0.00012 & 0.0009 \\
\hline Empme $^{2}$ & $(0.0007)^{* *}$ & $\begin{array}{l}(0.0007)^{* * *} \\
-.00000005 \\
(0.00000003)^{*}\end{array}$ & $(0.00007)^{*}$ & $\begin{array}{l}(0.00018)^{* * * *} \\
-0.000000024 \\
(0.00000006)^{* * *}\end{array}$ \\
\hline Fiold & $-0.01(0.01)$ & $-0.01(0.01)$ & $0.50(0.11)$ & $-0.34(0.86)$ \\
\hline Constant & $-4.32(0.84)^{* * *}$ & $-4.32(0.84)^{* * *}$ & $1.36(1.30)$ & $1.93(0.93)^{* *}$ \\
\hline Chi quadrado & $91.32 * * *$ & $91.48^{* * *}$ & & \\
\hline Sigma & & & $1.61(0.25)^{* * *}$ & $1.28(0.17)^{* * *}$ \\
\hline Nagelkerke $\mathrm{R}^{2}$ & 0.51 & 0.51 & & \\
\hline $\begin{array}{l}\text { Número de } \\
\text { Observações }\end{array}$ & 202 & 202 & 62 & 62 \\
\hline
\end{tabular}

Erro padrão em parênteses. *, **, *** Significante em $10 \%, 5 \%$ e 1\%, respectivamente. 
A Tabela 2 mostra que, em geral, os dois modelos apresentam um conjunto de coeficientes estatisticamente significativos, em termos de distribuição do Chiquadrado para o Logit e Sigma para o truncado. Os resultados confirmam a maioria das hipóteses previamente formuladas e, em particular, confirmam que existem importantes diferenças, em termos de variáveis determinantes, entre a propensão para inovar e intensidade inovativa de empresas da IBA. Os resultados dos testes das hipóteses e as diferenças na influência das variáveis sobre a propensão e a intensidade de inovações na IBA, serão reportadas e discutidas abaixo, iniciando com o primeiro modelo.

A estimativa do modelo Logit corretamente prediz $81 \%$ dos possíveis resultados (inovar/não inovar). Essa porcentagem de acerto do modelo é superior tanto à aposta ingênua de que 50\% das firmas inovam, quanto à aposta racional de que 73\% das empresas são não inovativas. Em adição, a estatística $\mathrm{R}^{2}$ de Nagelkerk indica que as variáveis selecionadas produzem uma melhora na predição da probabilidade de uma empresa ser inovativa. Por sua vez, a estatística Wald revela que a constante é estatisticamente significativa no nível de 1\%. Esses resultados fornecem suporte para a aceitação do modelo.

Em termos da influência particular de variáveis, a primeira equação confirma que a natureza incremental e a predominância de fontes externas de inovações colocam o investimento em tecnologias desenvolvidas externamente (tecmo) como a variável estatisticamente mais expressiva, numa estrutura multivariada, da propensão de uma empresa na IBA vir a inovar. Especificamente, dado que o antilog de 1.10 é 3,01, este investimento eleva em mais de três vezes as chances de uma empresa na IBA inovar ${ }^{(10)}$. Outra razão para este resultado é a existência na indústria de tecnologias redundantes (Cabral, 1999). Tecnologia redundante, de acordo com Padberg e Westgreen (1979), significa que o estado da arte em ciência e tecnologia de alimentos oferece oportunidades relevantes para inovação tecnológica na indústria de alimentos, em particular de determinados países. Em outras palavras, o estoque de tecnologias disponíveis apresenta um "superávit" comparado com o de tecnologias em uso na indústria.

A primeira equação também confirma a hipótese de que a probabilidade de uma empresa inovar está relacionada com o seu tamanho (empme). No entanto, embora a hipótese tenha sido confirmada com significância estatística, o coeficiente estimado é bastante pequeno.

A variável investimentos em $\mathrm{P} \& \mathrm{D}$ executado internamente (redmo) apresenta coeficiente relativamente expressivo e altamente significativo. Ele é significativo em nível de menos de 5\% e, dado que o antilog de 0,47 é superior a 1,5, eleva em mais de uma vez e meia as chances de uma empresa na IBA vir a inovar. Adicionalmente, a variável relativa a alianças (altot) para executar atividades 
externas de $\mathrm{P} \& \mathrm{D}$ também apresentou coeficiente estatisticamente significativo sobre a propensão para inovar na IBA. Estes resultados sugerem a rejeição da hipótese de que $P \& D$ não apresenta significativo relacionamento com inovação na indústria de alimentos e a aceitação da hipótese de associação entre alianças externas e inovatividade. Estes resultados confirmam as propostas de uma crescente importância para o papel de atividades terceirizadas de P\&D no processo de inovação de empresas alimentícias. Também sugere que, embora não dependendo exclusivamente de $\mathrm{P} \& \mathrm{D}$, a atividade de inovação na indústria pode ser alavancada com a competência em $\mathrm{P} \& \mathrm{D}$.

Com relação às demais variáveis na primeira equação, todos os resultados, à exceção de idade, confirmaram as hipóteses da pesquisa. O nível de propaganda (ADVMO) e o nível de funções na cadeia alimentar (PROTO) apresentaram coeficientes positivos, embora não significativos. Da mesma forma, embora em direção oposta, os níveis de exportações (EXPMO), de diversificação horizontal (ATOTA) e idade (FIOLD) apresentaram uma relação inversa com propensão para inovar. Estes resultados acrescentam evidências para as proposições teóricas de que a probabilidade de empresas alimentares inovarem não é significativamente determinada por ativos complementares, em termos de orientação para o mercado, integração vertical e diversificação horizontal. Apesar de mudanças na demanda, as empresas alimentares brasileiras continuam a ser orientadas para a produção.

A segunda equação do modelo Logit incluiu o termo quadrático da variável tamanho $\left(\mathrm{EMPME}^{2}\right)$. Como hipotetizado, o termo apresenta um coeficiente negativo e significativo $(\mathrm{p}<0,1)$. Então, confirma-se que a influência do tamanho não é linear, sugerindo que a probabilidade de inovar eleva-se com tamanho até um limite e então declina, sugerindo um relacionamento tipo ' $U$ ' invertido.

Por sua vez, o modelo Truncado foi incluído na análise para identificar as variáveis que afetam a maior intensidade de inovações em empresas na IBA, uma vez que elas tenham inovado. A premissa básica foi de que não existe razão para esperar que sejam similares as variáveis que influenciam a probabilidade de inovar e a intensidade de inovações. A existência de diferenças é confirmada pelos resultados. Do modelo Logit (primeira equação), apenas as variáveis relacionadas a tamanho de empresas e alianças novamente apresentaram, no modelo truncado (terceira equação), coeficientes positivos e significativos. Adicionalmente, a variável gastos com propaganda (advmo), como hipotetizado, apresentou significância estatística. Estes resultados sugerem que tamanho de empresa, alianças externas para desenvolver pesquisas e orientação para o mercado (expmo muda o sinal de negativo para positivo, embora não significativo) elevam a intensidade inovativa de empresas na IBA. 
Finalmente, a quarta equação reforça o resultado de que a influência de tamanho sobre atividades inovativas não é linear, desde que o coeficiente do termo quadrático de emprego $\left(\mathrm{EMPME}^{2}\right)$ seja negativo e estatisticamente significativo. Isto fornece evidência adicional de que não existe destacado efeito de escala sobre atividade inovativa (ver Klevorick, Levin, Nelson, \& Winter, 1995, p. 786).

\section{Considerações Finais}

Os resultados deste estudo mostram que o investimento em tecnologia (máquinas, equipamentos, processos e produtos) desenvolvida externamente à empresa é uma variável significativamente relacionada à propensão de empresas na IBA inovarem, confirmando as propostas de Connor et al. (1985) e os resultados empíricos apresentados por Galizzi e Venturini (1996). Três aspectos principais explicam esta relação: a natureza incremental das inovações, a predominância de inovações em tecnologias de capital e a existência de tecnologia 'redundante'. Portanto, isto está de acordo com a caracterização das empresas alimentares como 'dominada por fornecedores' (supplier-dominated) em termos de inovação. No entanto, este fator não é significativo na explicação da intensidade inovativa nestas empresas. Neste caso, outros fatores se destacam na elevação da eficiência inovativa, tais como orientação para o mercado e alianças para execução externa de $\mathrm{P} \& \mathrm{D}$, visando ao desenvolvimento de inovações.

Os resultados indicam também que o esforço interno das empresas em P\&D está relacionado com a probabilidade de uma empresa inovar, mas não com a intensidade de inovações. Este resultado foi o mais surpreendente da pesquisa, dadas as proposições prévias de que, enquanto uma empresa pode inovar sem se engajar em P\&D, a competência para inovar será elevada, se ela passar a desenvolver esta atividade (exemplos Cohen \& Levinthal, 1989; Connor et al., 1985).

Por sua vez, a variável tamanho de empresa foi confirmada como determinante muito importante tanto da propensão para inovar, quanto da intensidade de inovações. No entanto, nas duas associações, a influência do tamanho eleva-se até um limite e depois declina, sugerindo um relacionamento na forma de ' $U$ ' invertido. Similarmente a influência de tamanho, o nível de terceirização de P\&D eleva-se não apenas com a propensão para inovar, como também com a intensidade de inovações. Este resultado confirma que, embora com pouca relação, a parceria de empresas alimentícias com instituições privadas e públicas de P\&D tem-se apresentado como muito efetiva em alavancar a atividade inovativa de empresas na IBA. 
Com relação aos ativos complementares, foi confirmada a associação entre gastos com propaganda e intensidade inovativa. Este resultado era previsto, devido ao fato de que propaganda está relacionada com inovação de produto que, por sua vez, está relacionada com maior nível de produção de inovações. Os outros indicadores de ativos complementares, cadeias de produtos (diversificação horizontal), funções na cadeia produtiva (integração vertical), exportações e idade, não se apresentaram como significativos determinantes seja da propensão para inovar, seja da intensidade de inovações. Uma dedução aceitável destes resultados é de que a especialização em produtos e em estágios de produção, juntamente com a predominância do mercado doméstico, impõem mais pressão sobre as empresas na IBA para inovarem.

Do exposto pode-se concluir que as diferentes variáveis que influenciam a propensão para inovar e a intensidade de atividade inovativa de empresas na IBA, de diferentes maneiras, confirmam que os recursos e capacidades das empresas podem ser corretamente imputados como os principais fatores que operam na definição das possibilidades de inovação em empresas da IBA. Em outras palavras, dos incentivos e restrições colocados pelo contexto, o comportamento das empresas adicionalmente restringe ou eleva a trajetória inovativa delas.

Os resultados analisados sugerem que, para definir a estratégia tecnológica mais efetiva, as empresas precisarão identificar suas possibilidades e limitações tecnológicas e elevar a incorporação tanto de ativos para inovação quanto os complementares (recursos e capacidades). A melhor estratégia inovativa para estas firmas parece ser a alocação de recursos financeiros dirigidos à atualização de equipamentos, aprender como usá-los efetivamente e fortalecer o vínculo com fornecedores à montante da indústria. Também levando em consideração que os fatores investimentos em tecnologia externa, esforço em P\&D, tamanho e orientação para o mercado explicam entre uma vez e meia e três vezes as chances de uma empresa inovar ou ser mais intensiva em inovações, as empresas alimentícias serão mais efetivas na atividade inovativa, se adotarem como estratégias a alavancagem de seus esforços em P\&D e seus vínculos com empresas, instituições, universidades ou agências de pesquisa, com uma visão sobre os "mecanismos de indução" que surgem das oportunidades tecnológicas e de mercado.

Por sua vez, os resultados relativos à dependência de tecnologias desenvolvidas externamente sugerem que políticas públicas, voltadas para elevar a inovatividade na IBA, devem inicialmente voltar-se para o estímulo do desenvolvimento e difusão de tecnologias de aplicação geral pelos fornecedores à montante dessa indústria. Políticas complementares devem incentivar as empresas na IBA para a alocação 
de recursos, visando ao desenvolvimento da capacidade de absorção e adaptação de tecnologias (artefatos e conhecimento; explícitos e tácitos) desenvolvidas pelos fornecedores, públicos ou privados, e competidores. Estas políticas podem ser diretas, através, por exemplo, do financiamento de pesquisas e subsídios à aquisição de tecnologias, e indireta, através da eliminação de barreiras institucionais à inovação, tais como barreiras alfandegárias à importação de tecnologias. Adicionalmente, os resultados desta pesquisa também indicam que incentivar as parcerias das empresas alimentícias para o desenvolvimento de projetos inovativos, alcançar uma escala mínima (tamanho) e elevar sua orientação para o mercado, deve conduzir a um grau superior de inovatividade (absoluta e relativa) na IBA.

\section{Artigo recebido em 28.10.2004. Aprovado em 11.03.2005.}

\section{Notas}

${ }^{1}$ Exemplo citado em Scherer, F. M., \& Ross, D. (1990). Industrial market structure and economic performance (p. 613). Boston: Houghton Mifflin Company, Baseado em: Smith, A. (1776). An inquiry into the nature and causes of the wealth of nations. Modern library (edition 1937), New York and Pratten, C. F. (1980). The manufacture of pins. Journal of Economic Literature, 18, 93-96.

${ }^{2}$ É importante esclarecer que uma vez que a definição de novo pode estar ligada à utilização em nível de mundo, país ou empresa, pode-se dizer que o trabalho contempla também o estágio de imitação. Porém, a ênfase será sobre inovação ou grupo de inovações geradas e/ou adotadas por uma empresa em uma indústria particular (no caso de alimentos) e não sobre como uma inovação em particular é originada e disseminada pela economia.

${ }^{3}$ Também é importante esclarecer que os autores citados, iniciando por Schumpeter, definem inovação num sentido mais amplo, incluindo inovações de materiais, mercados ou organizacionais. No entanto,o objeto deste trabalho é a inovação tecnológica (produto ou processo). Em diante, inovação e inovação tecnológica serão utilizados com o mesmo sentido.

${ }^{4}$ Reconhece-se que este é um número limitado de indicadores para mensurar o construto. Kohli, A. K., Jaworski, B. J., \& Kumar, A. (1993). MARKOR: a measure of market orientation. Journal of Marketing Research, 30(4), 467-477, por exemplo, propõem a mensuração de orientação para o mercado (MARKOR), a partir de um conjunto de 32 indicadores (escalar).

${ }^{5}$ Ver.Teece, D. (1988). Technological change and the nature of the firm. In G. Dosi, C. Freeman, R. R. Nelson, G. Silveberg, \& L. Soete (Eds.). Technical change and economic theory (p. 268). London: Pinter Publishers, sobre estes dois tipos de inovações.

${ }^{6}$ Patel, P., \& Pavitt, K. (1995). Patterns of technological activity: their measurement and interpretation. In P. Stoneman (Ed.). Handbook of the economics of innovation and technological change. Oxfor: Blackwell, e Santarelli, E., \& Piergiovanni, R. (1996). Analyzing literature-based innovation output indicators: the italian experience. Research Policy, 25(5), 689-711. apresentam uma extensa lista de vantagens e desvantagens (limitações) dos indicadores de inovações. Cabral (1999) resume estes aspectos de patentes e P\&D. 
${ }^{7}$ Este sentido amplo foi adotado baseado na premissa de que inovações tecnológicas em nível mundial não constituem um evento muito comum. Além do mais, como Rosegger, G. (1996). The economics of production and innovation: an industrial perspective (p. 172). Oxford: Butterworth-Heinemann Ltd. afirma "um produto ou processo dificilmente permanece imutável em sua difusão; portanto, "primeiras" repetem-se após o surgimento da inovação, e muitas delas vêm a ser tão significativas, técnica e economicamente, quanto a inovação base". Por sua vez, Kline, S. J., \& Rosenberg, N. (1986). An overview of innovation. In S. J. Kline \& N. Rosenber (Eds.). The positive sum strategy: harnessing technology for economic growth (p. 283). Washington, DC: National Academy Press, sugerem que as "inovações mais importantes passam por mudanças drásticas em seu ciclo de vida; mudanças que podem, como ocorre frequentemente, transformar totalmente sua significância econômica". Finalmente, outra razão importante para esta decisão foi seguir estudos semelhantes em outros países e comparar os resultados. Portanto, este trabalho está considerando tanto o processo de inovação, quanto o de imitação em termos Schumpeterianos.

${ }^{8}$ IBGE - Instituto Brasileiro de Geografia e Estatística: Resolução do Presidente N 054/94. Esta classificação é bastante similar a NACE, SIC Inglês e Norte-Americano de 1992.

${ }^{9}$ Dos 43.034 estabelecimentos. Esta pequena diferença confirma que a maioria das empresas alimentícias desenvolve suas atividades em um só local.

${ }^{10}$ Como os coeficientes são expressos em logaritmos, eles precisam ser transformados através do cálculo do antilog de modo que o efeito relativo sobre as possibilidades (chances) de o evento acontecer possam ser avaliados diretamente (Ver Hair Jr., J. F., Andersos, R. E., Tatham, R. L., \& Black, W. C. (1998). Multivariate data analysis. Upper Sadle River, NJ: Prentice-Hall).

\section{Agradecimento}

O autor agradece aos revisores anônimos do artigo pelas excelentes sugestões ao texto original.

\section{REFERÊNCIAS BibLIOGRÁfICAS}

Braga, H., \&

Wilmore, L. (1991).

Technological imports and technological effort: an analysis of their determinants in Brazilian firms. The Journal of Industrial Economics, 39(4), 421-432.

Burton, M.,

Tomlinson, M., \&

Young, T. (1994).

Consumers' decision whether or not to purchase meat: a double hurdle analysis of single households. Journal of Agricultural Economics, 45(2), 202-212.

Cabral, J. E. O. (1999).

Patterns and determinants of technological innovation in the Brazilian food industry. PhD. Thesis, The University of Reading, Reading, UK. 
Cesaratto, S., \&

Mangano, S. (1993).

Technological profiles and economic performance in the italian manufacturing sector. Economics of Innovation and New Technology, 2(3), 237-256.

Christensen, J. F. (1995).

Asset profiles for technological innovation. Research Policy, 24(5), 727-745.

Christensen, J. F. (1996).

Innovative assets and inter-asset linkages: a resource-based approach to innovation. Economics of Innovation and New Technology, 4(3), 193-209.

Christensen, J. F.,

Rama, R., \&

Von Tunzelmann, N. (1996).

Industry studies of innovation using CIS data: study on innovation in the european food products and beverages industry. CSIC Espanha. Recuperado em 9 junho, 1999, de http:/ / www.ieg.csic.es/cv/docs/ EUREPORT.pdf

Cohen, J. M., \&

Levinthal, D. A. (1989).

Innovation and learning: the two faces of R\&D. The Economic Journal, 99(397), 569-596.

Connor, J. M.,

Rogers, R. T.,

Marion, B. W., \&

Mueller, W. F. (1985).

The food manufacturing industries. Lexinton-MA: Lexington books.
Freeman, C. (1982).

The economics of industrial innovation. Londres: Frances Printer.

Freeman, C., \&

Soete, L. (1997).

The economics of industrial innovation. London: Pinter.

Galizzi, G., \&

Venturini, L. (1996).

Product innovation in the food industry: nature, characteristics and determinants. In G. Galizzin \& L. Venturini (Eds.). Economics of innovation: the case of food industry. Hedelberg: Physica-Verlag.

Goetz, S. J. (1995).

Markets, transaction cost, and selectivity models. In G. J. Scott (Ed.). Economic development in prices, products and people: analysis agricultural markets in developing countries, Boulder-CO: Lynne Riemer Publishers.

Grunert, K.G.,

Clerk, F.,

Goransson, G.,

Harmsen, H.,

Kuiper, E.,

Meulenberg, M.,

Ottowitz, T., \&

Traill, B. (1995).

Innovation in the food sector: between technology-push and demand-pull (EU AAIR programme). (Discussion Paper Series n.10), University of Reading, Reading, UK.

Huiban, J., \&

Boushina, Z. (1998).

Innovation and the quality of labour factor: an empirical investigation in the French food industry. Small Business Economics, 10(4), 389-400. 
Klevorick, A. K.,

Levin, R. C.,

Nelson, R. R., \&

Winter, S. G. (1995).

On the sources and significance of interindustry differences in technological opportunities. Research Policy, 24(2), 185-205.

Myers, S., \&

Marquis, D. G. (1969).

Successful industrial innovations: a study of factors underlying innovation in selected firms. Washington, DC: National Science Foundation.

Nelson, R. R. (1959).

The simple economis of basic scientific research. Journal of Political Economy, 61, 297-306.

Padberg, D. I., \&

Westgren, R. E. (1979).

Product competition and consumer behavior in the food industries. American Journal of Agricultural Economics, 67(4), 620-626.

Pavitt, K. (1984).

Sectoral patterns of technical change: towards a taxonomy and theory. Research Policy, 13(6), 343-373.

Scherer, F. M. (1965).

Size of firm, oligopoly, and research: a comment. Canadian Journal of Economics and Political Science, 31(2), 256-66.

Schumpeter, J. A. (1939).

Business cycles: a theoretical, historical, and statistical analysis of the capitalist process. Philadelphia: Porcupine Press, Inc.

Smith, A. (1776).

An inquiry into the nature and causes of the wealth of nations. New York: Modern Library. (First edition 1937).

Teece, D. (1986).

Profiting from technological innovation: implications for integration, collaboration, licensing and public policy. Research Policy, 15(6), 285-306.

Teece, D. (1996).

Firm organization, industrial structure, and technological innovation. Journal of Economic Behavior and Organization, 31(2), 193-224.

Toffler, A. (1970).

Future shock. London: The Bodley Head.

Wakelin, K. (1998).

Innovation and export behavior at the firm level. Research Policy, 26(7-8), 829-841. 